\title{
Common Origin of Brachiocephalic and Left Common Carotid Arteries: Proposal of New Terminology
}

$\mathbf{T}$ here is recent renewed interest in studying the aortic arch variants, fueled, at least in part, by suggestions that they might play a role in aortic pathologies. ${ }^{1,2}$ The human aortic arch has several branching patterns, reflecting its complex embryologic development. The most common or the standard is a 3-vessel pattern (Fig $1 A$ ), with a reported prevalence between $65 \%$ and $86 \%{ }^{3}$; the first branch is the brachiocephalic trunk, followed by the left common carotid artery and then the left subclavian artery.

The most common aortic arch variant by far is a 2 -vessel branching pattern: The first branch is a common origin of the brachiocephalic trunk and left common carotid artery, and the second branch is the left subclavian artery (Fig 1B), or, less commonly, the left common carotid artery takes origin from the brachiocephalic trunk. ${ }^{1}$ The reported prevalence of this variant is between $7 \%$ and 26\%, with the highest prevalence in African and South American populations. ${ }^{3}$ This variant has been historically termed the "bovine" arch, yet the origin of this term is not clear. ${ }^{4}$ Despite several critiques, ${ }^{4,5}$ this term is still in wide use in radiologic and surgical reports and journal publications, ${ }^{1,2}$ mainly because it is familiar and shorter than the full anatomic description. Another term, the "ovine arch," is used in some institutions to describe the same variant, though far less commonly. ${ }^{5}$ Both terms are known to be misnomers, and this aortic arch variant is not the typical aortic branching pattern in cattle or sheep. ${ }^{4,5}$

The "bicarotid trunk/truncus bicaroticus" is a third name for the common origin of the brachiocephalic trunk and left common carotid artery. ${ }^{6}$ However, in our opinion, this term should be reserved for other 3-vessel branching variants in which there is a separate origin of a normal site or an aberrant right subclavian artery in combination with a common trunk for both carotid arteries and a separate origin for the left subclavian artery (Fig 1C).

A fourth name for the same variant, the "common brachiocephalic trunk," is sometimes used. This term is short but less popular and is clearly anatomically deficient. Last, a fifth name was proposed, "common origin of innominate and carotid arteries," 5 which is not a misnomer but is still long and lacks familiarity.

Moreover, the association of this variant with a separate aortic origin of the left vertebral artery constitutes a different branching variant, which was termed a combined "bovine" arch and direct origin of the left vertebral artery, ${ }^{3}$ despite being a 3- rather than the 2-vessel branching pattern suggested by the term "bovine" (Fig 1D). Another rare variant that we recently encountered is a right aortic arch with a common origin of the left brachiocephalic trunk and right common carotid artery, causing confusion about the terminology and raising the question of whether it could be termed a "reverse bovine" arch (Fig 1E).

To summarize, the most common variant of the human aortic arch branching pattern has 5 different confusing terms in use. Therefore, we propose naming the common origin of the brachiocephalic trunk and left common carotid artery the "brachio-bicephalic" trunk. This term is short enough and more anatomically correct, with familiarity and resemblance to the original name of the brachiocephalic trunk. Additionally, it is appropriate to use when combined with a direct origin of the left vertebral artery and could be labeled "left" when this variant is present in a right-sided aortic arch.

\section{REFERENCES}

1. Hornick M, Moomiaie R, Mojibian H, et al. 'Bovine' aortic arch: a marker for thoracic aortic disease. Cardiology 2012;123:116-24 CrossRef Medline

2. Moorehead PA, Kim AH, Miller CP, et al. Prevalence of bovine aortic arch configuration in adult patients with and without thoracic aortic pathology. Ann Vasc Surg 2016;30:132-37 CrossRef Medline

3. Popieluszko P, Henry BM, Sanna B, et al. A systematic review and meta-analysis of variations in branching patterns of the adult aortic arch. J Vasc Surg 2017 Aug 30. pii: S0741-5214(17)31788-3 [Epub ahead of print] CrossRef Medline

4. Layton KF, Kallmes DF, Cloft HJ, et al. Bovine aortic arch variant in humans: clarification of a common misnomer. AJNR Am J Neuroradiol 2006;27:1541-42 Medline

5. Katz JC, Chakravarti S, Ko HH, et al. Common origin of the innominate and carotid arteries: prevalence, nomenclature, and surgical implications. J Am Soc Echocardiogr 2006;19:1446-48 CrossRef Medline

6. Reinshagen L, Vodiskar J, Mühler E, et al. Bicarotid trunk: how much is "not uncommon"? Ann Thorac Surg 2014;97:945-49 CrossRef Medline

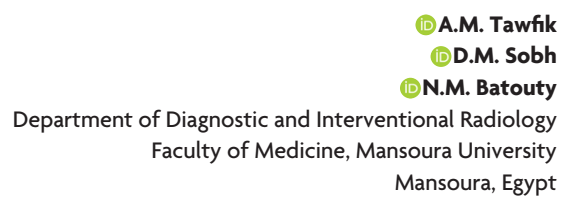



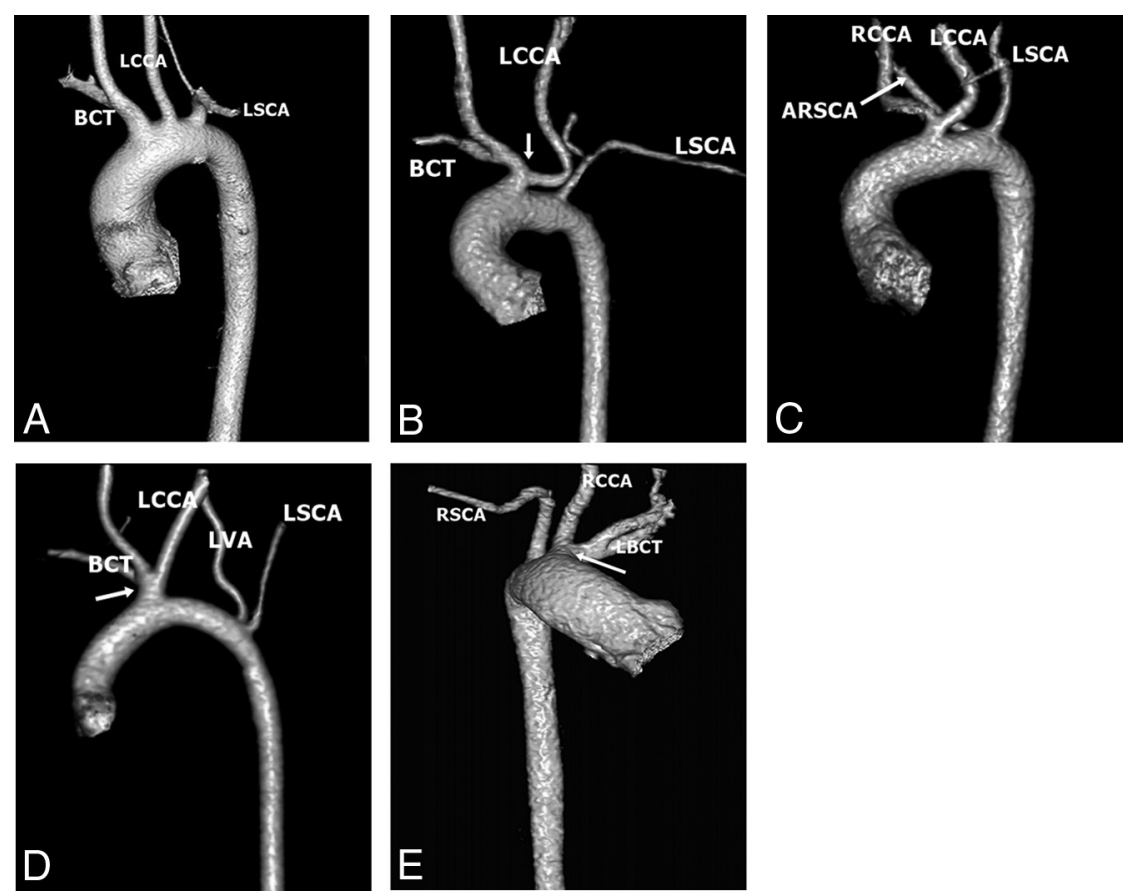

C

FIG 1. Volume-rendered CT of some aortic arch variants. A, Left aortic arch with normal branching pattern. $B$, Left aortic arch with a common origin (arrow) of the brachiocephalic trunk and left common carotid artery. C, Left aortic arch with separate origins of an aberrant right subclavian (arrow), and the left subclavian arteries and a common trunk for both carotid arteries. $D$, Left aortic arch with a common origin (arrow) of the brachiocephalic trunk and left common carotid artery and a separate origin of the left vertebral and left subclavian arteries. E, Right aortic arch with a common origin (arrow) of the left brachiocephalic trunk and the right common carotid artery. LCCA indicates left common carotid artery; RSCA, right subclavian artery; LSCA, left subclavian artery; RCCA, right common carotid artery; LBCT, left brachiocephalic trunk; ARSCA, aberrant right subclavian artery; LVA, left vertebral artery; BCT, brachiocephalic trunk. 